\title{
Influence of Desorption of Gases from the Anode on the Reading of Pressure Determined by Field Emission Microscopy
}

\author{
Tatsuo IWATA and Kap-Soon CHANG* \\ Department of Electronic and Information Technology, School of Engineering, \\ Hokkaido Tokai University \\ 5-1-1-1 Minaminosawa, Minamiku, Sapporo 005, JAPAN \\ ${ }^{*}$ Department of Electronics, Faculty of Engineering, Tokai University \\ 117 kitakaname, Hiratuka, Kanagawa 259-12, JAPAN
}

(Received April 20, 1994, Accepted July 2, 1994)

\begin{abstract}
Outgassing rate upon electron impact from the phosphor screen of a field emission microscope has been measured as a function of anode current and applied voltage. The outgassing rate increased quickly with anode voltage up to about $1.5 \mathrm{kV}$; after that it increased only slightly. At the practical anode voltage used in field emission experiments, the outgassing rate substantially depended on only emission current and was found to be $3 \times 10^{-1} I_{\mathrm{e}}\left(\mathrm{Pa} \cdot \mathrm{m}^{3} / \mathrm{s} \cdot \mathrm{m}^{2}\right)$. From this relationship, we conclude that if we use a $\ln \mathrm{A}$ or lower emission current there are few effects on the reading of pressure with a field emission vacuum gauge in UHV, and in XHV as well.
\end{abstract}

\section{Introduction}

Decrease in field emission current from an initially cleaned field-emission tip at constant anode voltage is a good indication of contamination by residual gases in vacuum systems. Since the amount of gas adsorbed on an emitter surface is proportional to the product of pressure and time, the time required for the same decrease in emission current or for the appearance of some characteristic emission pattern should be inversely proportional to the pressure. Based on the relationship between the time and the pressure, several authors have proposed methods to determine the pressure by field emission microscopy $\left(\right.$ FEM) ${ }^{1) \sim 5)}$. These methods could detect a very low pressure far below the X-ray limit of the conventional Bayard-Alprt gauge. Because these methods essentially measure the integrated amount of adsorbate, we can determine only the average pressure of the measured interval.

Recently, we have developed new operation modes that make it possible to carry out real-time measurement of the pressure from either the changing rate of the emission current at a constant applied voltage $\left.{ }^{6}\right)$ or from the changing rate of the anode voltage for a constant emission current ${ }^{7}$, but many attractive features ${ }^{3)}$ of FEM gauge are still effective. One of the aims of this study is to elucidate the practical operation condition suitable for these new operation modes of the FEM pressure gauge.

Irrespective of the operation mode, the gas evolution caused by electron impact increases the pressure of the vacuum system to a greater or lesser extent when the FEM is operated. The emission current must be reduced to as low as possible to minimize gas evolution from the screen. On the contrary, adequate emission current must be maintained for accurate determination of the pressure because low noise signals of the emission current are needed for precision signal processing. To minimize the effect, some authors ${ }^{8)}$ introduced a metallic anode which can be thoroughly degassed. This type of anode cannot be used for applications that require observation of the emission pattern which gives information on adsorbed species. It is sometimes very important, for studies of surface phenomena, to know the major components of chemically active gases; therefore, the outgassing rate of the phosphor screen is still an essential factor when the FEM is used as a pressure gauge.

As far as we know, in the literature there are no reports on the outgassing rate under practical operation conditions of FEM; therefore, we must experimentally elucidate the practical operation conditions which satisfy these contradictory requirements. In this study, the amount of desorbed gases from the phosphor screen of a conventional FEM 


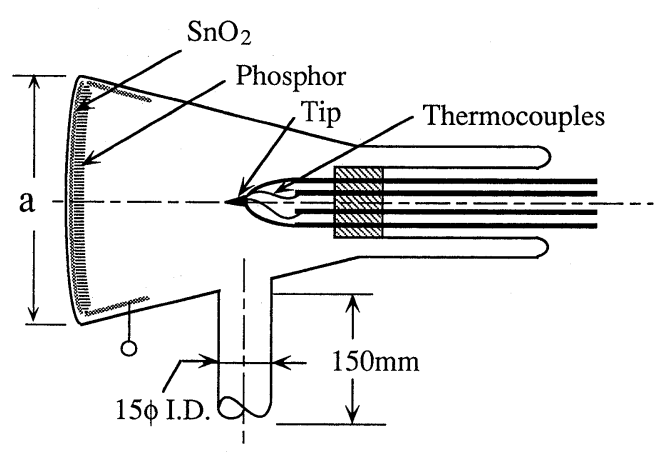

Fig. 1 Schematic drawing of the FEM. Screen diameter a is $8 \mathrm{~cm}$ for the head prepared by nitrocellulose method and $10 \mathrm{~cm}$ for the head prepared by phosphoric-acid method.

has been measured as a function of emission current and anode voltage.

\section{Experimental}

A schematic drawing of the FEM head made of Pyrex glass is shown in Fig. 1. Two different screens having diameters of 8 and $10 \mathrm{~cm}$ were used. The phosphor screens were prepared in two different ways by the mitrocellulose method and the phosphoric acid method, both of which were presented by Gomer'). These microscope heads were connected to vacuum systems with a tube. The conductance of the tube was about $2 l / \mathrm{s}$ for nitrogen. Two vacuum systems were used. One of them included a $160 \mathrm{l} / \mathrm{s}$ ion pump and tubing of 60 $l / \mathrm{s}$. The other system included a $20 \mathrm{l} / \mathrm{s}$ ion pump and tubing of $20 \mathrm{l} / \mathrm{s}$. Conventional glass-enveloped Bayard-Alpert gauges with sensitivity of 12 Torr $^{-1}$ were used as a standard. A schematic drawing of the vacuum system is presented in Fig. 2. Ultimate pressure of the systems, about $4 \times 10^{-9} \mathrm{~Pa}$, was attained after bake out at $400^{\circ} \mathrm{C}$ for 8 hours.

The outgassing rate $Q$ of electron stimulated desorption (ESD) was calculated by

$$
Q=P \cdot S / A_{\text {screen }}
$$

where $P$ is the pressure measured by the Bayard-Alpert gauge, $S$ is the effective pumping speed at the measured point and $A_{\text {screen }}$ is the area of the phosphor screen.

The constitution of residual gas was analyzed by a quadrupole mass filter (not shown in Fig. 2), and $\mathrm{H}_{2}, \mathrm{O}, \mathrm{CO}$ or $\mathrm{N}_{2}$ or both $(\mathrm{m} / \mathrm{e}=28)$ and a small amount of $\mathrm{CO}_{2}$ were detected.

A rhenium field emission tip was mounted near the top of the U-shaped tungsten supporting loop.

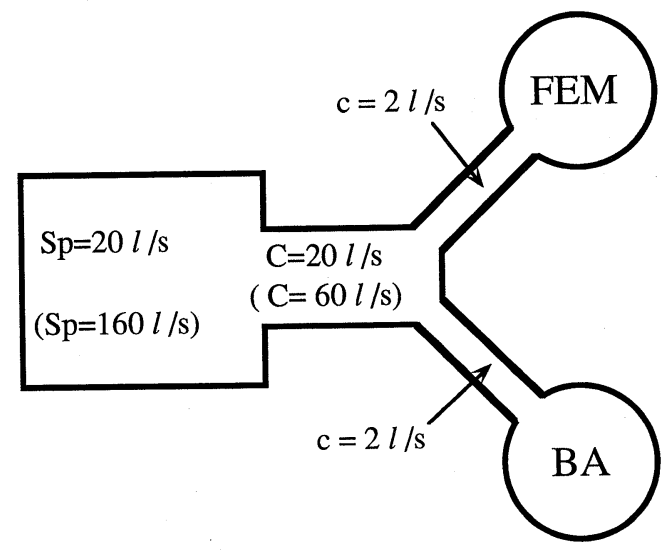

Fig. 2 Schematic drawing of the vacuum system used. One system includes a $20 \mathrm{l} / \mathrm{s}$ ionpump and $20 \mathrm{l} / \mathrm{s}$ tubing. Another includes a $160 \mathrm{l} / \mathrm{s}$ ionpump, $60 \mathrm{l} / \mathrm{s}$ tubing and quadrupole mass filter (not shown).

The tip was heated by ac resistive heating of the tungsten supporting loop and the temperature was measured by $\mathrm{W}-5 \% \mathrm{Re} / \mathrm{W}-26 \% \mathrm{Re}$ thermocouples at the vicinity of the tip.

\section{Results and Discussion}

\subsection{Dependence of gas evolution on anode vol- tage}

Although anode voltage in our setup cannot be adjusted without change in emission current, thermal-field emission was adopted to maintain a constant anode current. In this case emission current can be controlled by temperature. Figure 3 shows the pressure measured by the Bayard-Alpert guage at the exit of the connecting tube of the FEM head as a function of anode voltage for the $20 \mathrm{l} / \mathrm{s}$ ionpump vacuum system. The current density at the phosphor screen is $1 \times 10^{-5} \mathrm{~A} / \mathrm{cm}^{2}$. The shape of the pressure vs anode voltage curve shows typical features of ESD, but the curve seems to be shifted toward higher acceleration voltage compared to the case of a typical metallic surface. It rather resembles the oxygen outgassing curve of glass reported by Dawson ${ }^{10}$. Based on this result, the charge-up of the phosphor layer is considered, though the screen is coated with a conductive $\mathrm{SnO}_{2}$ layer.

Thermal desorption of gases by surface heating produced by electron bombardment becomes predominant as the power density on the anode increases. At the anode voltage above $1 \mathrm{kV}$, where the power density is about $10 \mathrm{~mW} / \mathrm{cm}^{2}$, thermal desorption may occur, therefore the gradual in- 


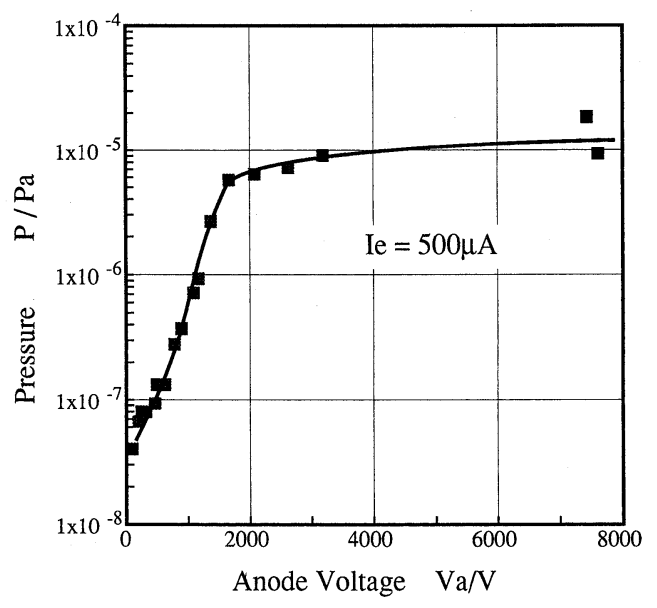

Fig. 3 Dependence of the outgassing rate of ESD from the phosphor screen prepared by the nitrocellulose method on the anode voltage.

crease in pressure with anode voltage seems partly due to the thermal effect in the present case. It is thought that the ESD of the phosphor screen is virtually independent of the voltage applied under a typical operation condition of the FEM gauge.

\subsection{Dependence of outgassing rate on emission} current

The outgassing rate of ESD from the phosphor screen was calculated from Eq. 1 as a function of anode current and is shown in Fig. 4. Here, the data corresponding to the anode voltage greater than $2 \mathrm{kV}$ were collected. The data for the screen prepared by the nitrocellulose method ( $\square)$ and by the phosphoric acid method $(\bullet)$ are shown. The outgassing rate, which is calculated from ultimate pressure by assuming that the pressure is dependent on ESD, is also plotted $(\diamond)$. The measured outgassing rate below the emission current of $1 x$ $10^{-5} \mathrm{~A}$ seems to deviate systematically. There are several possible reasons for the deterioratio of the accuracy of measurement for a small emission current and resulting small increase in pressure. One reason may be related to the method of determining the increase in the pressure. Here, we calculate the increase in pressure by subtract the background pressure from the measured pressure. The errors introduced become greater as the increase in pressure smaller. Another important and more plausible possibility is the pressure dependence of the pumping speed of ion pumps used. It is well known that the pumping speed of a ion pump below $10^{-7} \mathrm{~Pa}$ is a function of the pressure. We did not compensate the pumpig speed to calculate the effective pumping speed because we had no reliable data of pump-

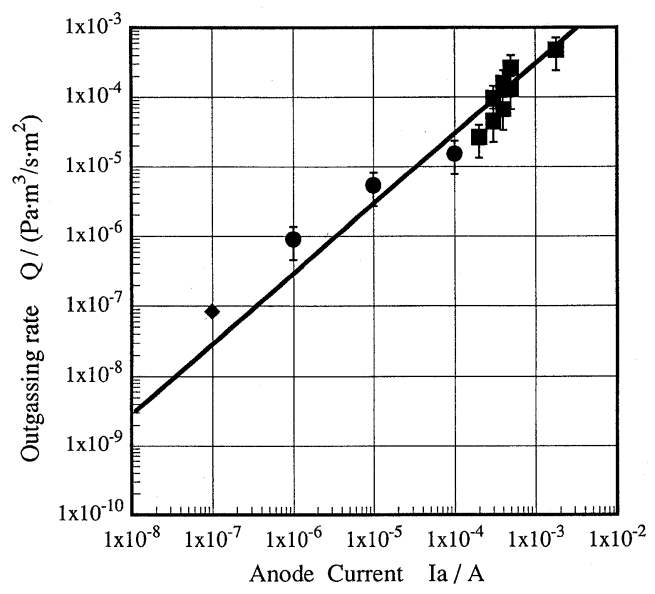

Fig. 4 Outgassing rate of ESD from the screen prepared by the nitrocellulose method ( $\square$ ) and by phosphoric-acid method $(\bullet)$ as a function of anode current. The mark $(\bullet)$ represents a value calculated at ultimate pressure assuming that the pressure is determined by ESD.

ing speed of ion pump at pressures below $10^{-8} \mathrm{~Pa}$. Therefore the calculated outgassing rate is expected to become larger compared to the true value as emission current decrease. If we assume a pumping speed of about a quarter of its nominal value for emission current of $1 \times 10^{-6} \mathrm{~A}$ and half of its nominal value for the emission current of $1 \times 10^{-5} \mathrm{~A}$, the measured outgassing rate well fits the strait line shown in Fig. 4. Decrease in pumping speed as expected above seems very probable, judging from the data reported ${ }^{11)}$. It is clear from the above considerations that the outgassing rate is directly proportional to the anode current and is almost independent of the screen preparation method. Thus, we can obtain the outgassing rate $Q=3 \times$ $10^{-1} I_{\mathrm{e}}\left(\mathrm{Pa} \cdot \mathrm{m}^{3} / \mathrm{s} \cdot \mathrm{m}^{2}\right)$ from the result.

Assuming that the current density is uniform throughout the phosphor screen, we can calculate the ESD yield of $6 \times 10^{-2}$ molecules/ electron. This value seems reasonable compared to the value found in the literature ${ }^{12}$. It is well known that the ESD yield is very sensitive to the surface condition; therefore, the value calculated above is not universal, but it still seems to be effective for determining the practical operation conditions of the FEM pressure gauge. It is predicted that when the FEM gauge is operated at emission current of $1 \mathrm{nA}$, pressure on the order of $10^{-10} \mathrm{~Pa}$ can be measured even in the case of the ESD yield ten times larger than the value determined in this work. The measurement of the current in the order of $10^{-9} \mathrm{~A}$ with 
modern electronic instruments is not very difficult; therefore, the phosphor-screen FEM is promising as a gauge of XHV.

\section{Conclusions}

The desorption rate of ESD from the phosphorscreen of a Pyrex-glass-enveloped FEM depended strongly on the energy of incident electrons up to about $1.5 \mathrm{kV}$, and was nearly independentof the energy above that. The outgassing rate, $Q$, for the acceleration voltage above $2 \mathrm{kV}$ was proportional to the anode current and was found to be $3 \times 10^{-1}$ $I_{\mathrm{e}}\left(\mathrm{Pa} \cdot \mathrm{m}^{3} / \mathrm{s} \cdot \mathrm{m}^{2}\right)$.

We measured the ultimate pressure of our vacuum system, $4 \times 10^{-9} \mathrm{~Pa}$, at the emission current of $0.1 \mu \mathrm{A}$. At the emission current acceptable for reliable measurement, for example, $1 \mathrm{nA}$, the outgassing rate was low enough to measure the pressure of UHV or XHV.

\section{Acknowledgment}

We would like to thank Mr. A. Yamamoto and Mr. N. Matsuo for help with the experiments and for assistance in preparing the manuscript.

\section{References}

1) V. Ya. Pliskovskii, M. S. Sukonnik, and V. A. Shishkin: Zh. Tekh. Fiz., 44 (1974) 2178. (Sov. Phys. Tech. Phys., 19 (1975) 1347.)

2) M. S. Sukonnik and V. A. Shishkin: Zh. Tekh. Fiz., 46 (1976) 863. (Sov. Phys. Tech. Phys., 21 (1976) 500.)

3) L. de Chernatony and J. Yarwood: Vacuum, 29 (1978) 125.

4) W. P. Dyke and J. K. Trolan: Phys. Rev., 84 (1953) 799.

5) C. Oshima, R. Souda, M. Aono, and Y. Ishizawa: Shinku (J. Vac. Soc. Jpn.), 26 (1983) 726.

6) T. Iwata and K.-S. Chang: Shinku (J. Vac. Soc. Jpn.), 36 (1993) 193.

7) T. Iwata and K.-S. Chang: Appl. Surf. Sci., 76/77 (1994) 31.

8) E. E. Martin, J. K. Trolan, and W. P. Dyke: J. Appl. Phys., 31 (1960) 782.

9) R. Gomer: Field Emission and Field Ionization (Harvard University Press, Cambridge, 1961).

10) P. HH. Dawson: Nuovo Cimento Suppl., 5 (1967) 612.

11) J. F. O'Hanlon: A User's Guide to Vacuum Technology (Wiley Interscience, New York, 1989).

12) M. J. Drinkwine and D. Lichman: Progress in Surf. Sci., 8 (1977) 123. 